\title{
Poly(4-vinylpyridinium)hydrogen sulfate: A novel and efficient catalyst for the synthesis of 13-aryl-indeno[1,2-b]naphtha[1,2-e]pyran-12(13H)-ones under solvent-free conditions
}

\author{
Majid Ghashang a, Syed Sheik Mansoor b,*, Krishnamoorthy Aswin b \\ a Faculty of Sciences, Najafabad Branch, Islamic Azad University, Najafabad, Esfahan, Iran \\ ${ }^{\mathrm{b}}$ Bioactive Organic Molecule Synthetic Unit, Research Department of Chemistry, C. Abdul Hakeem College, Melvisharam - 632 509, Tamil Nadu, India
}

\section{A R T I C L E I N F O}

\section{Article history:}

Received 5 August 2013

Accepted 2 September 2013

Published 20 January 2014

\section{Keywords:}

Poly(4-vinylpyridinium)hydrogen

sulfate

Indenonaphthopyrans

$2 H$-indene-1,3-dione

$\beta$-Naphthol

\begin{abstract}
A B S T R A C T
An facile and efficient protocol for the synthesis of 13-aryl-indeno[1,2-b]naphtha[1,2-e]pyran$12(13 H)$-ones has been developed that proceeds via the one-pot three-component sequential reaction of an aromatic aldehyde with $\beta$-naphthol and $2 H$-indene-1,3-dione under solvent-free conditions in the presence of a poly(4-vinylpyridinium)hydrogen sulfate $\left(\mathrm{P}(4-\mathrm{VPH}) \mathrm{HSO}_{4}\right)$ catalyst. The catalyst can be reused several times, making this procedure facile, practical, and sustainable. The simple experimental procedure, solvent-free reaction conditions, use of an inexpensive catalyst, short react time, and excellent yields are some of the major advantages of this methodology.
\end{abstract}

(C) 2014, Dalian Institute of Chemical Physics, Chinese Academy of Sciences. Published by Elsevier B.V. All rights reserved.

\section{Introduction}

Multicomponent reactions (MCRs) are an important class of convergent organic reactions, where three or more starting materials react to form a product that contains atoms derived from all of the participating reagents, and these processes are often referred to as high atom economy. MCRs have been widely used for the convergent synthesis of complex and important organic molecules from simple and readily available starting materials and have emerged as a powerful tool for drug discovery. This approach offers the possibility of producing large numbers of compounds with the same scaffold and a diverse range of side chains, and therefore allows for fast and efficient structural optimization with respect to the activity and selectivity properties of a pharmaceutically interesting com- pound series, as well as enabling the evaluation of several other pharmacologically important features of an active molecule [1-3].

In recent years, the synthesis of naphthopyran-based compounds and their derivatives has received an increasing amount of attention because of their biological activity, such as their antiviral [4], mutagenic [5], antimicrobial [6], antiproliferative [7], and anticancer activity [8], as well as their activity toward the central nervous system [9]. Indenopyrans are 'privileged medicinal scaffolds' that have been used for the development of pharmaceutical agents in a variety of different disease areas. Compounds with this particular motif show a wide range of pharmacological activity, such as antiulcer [10], antiallergenic [11], and antidepressant activity [12]. Indenopyrans have received increasing levels of attention in recent years

\footnotetext{
* Corresponding author. Tel: +91-04172-266487; Fax: +91-04172-266487; E-mail: smansoors2000@yahoo.co.in 
because of their pharmacological and medicinal importance.

A survey of the literature revealed that only few methods are available for the synthesis of indenonaphthapyrans. Wu et al. [13] developed a simple and facile procedure for the synthesis of 13-aryl-indeno[1,2- $b]$ naphtha[1,2-e]pyran-12(13H)-ones by the one-pot condensation of $\beta$-naphthol with an aldehyde and $2 \mathrm{H}$-indene-1,3-dione under solvent-free conditions in the presence of silica chloride as a heterogeneous catalyst. Wu et al. [14] also reported the synthesis of 13-aryl-indeno[1,2-b]naphtha[1,2-e]pyran-12(13H)-ones under solvent-free conditions using sulfamic acid as a catalyst. However, there are several limitations to these methods, such as the requirement for forcing reaction conditions, unsatisfactory yields, and long reaction time. Furthermore, some of these catalysts are either expensive or difficult to prepare. Given the importance of indenonaphthopyrans and the urgent need for the development of environmentally benign chemical production processes, the development of suitable green synthetic methods for these compounds has attracted considerable interest.

Solid acids have many advantages over liquid acids in terms of their application in organic catalysis. For example, solids acids do less harm to the environment and do not cause any significant issues in terms of their corrosion or the disposal of effluent containing these materials. Furthermore, they are reusable and readily separated from liquid products. As economically and ecologically benign catalysts, research toward their application has attracted considerable interest from academia and industry, and the replacement of traditional homogeneous catalysts with solid acids appears to be becoming an inevitable trend [15].

Poly(4-vinylpyridinium) hydrogen sulfate ( $\left.\mathrm{P}(4-\mathrm{VPH}) \mathrm{HSO}_{4}\right)$ has recently received considerable attention as an efficient catalyst for the construction of carbon-carbon and carbon-hetero atom bonds [15-18] because of its eco-friendly nature, ease of handling, high reactivity, and easy work-up procedures. $\mathrm{P}(4-\mathrm{VPH}) \mathrm{HSO}_{4}$ has been reported as a novel solid acid catalyst for the chemoselective 1,1-diacetate protection and deprotection of aldehydes [15], as well as the synthesis of 14-aryl-14Hdibenzo[a,j]xanthenes [16], xanthene derivatives [17], and 12aryl- $12 H$-indeno[1,2- $b]$ naphtho[3,2-e]pyran-5,11,13-triones [18].

In continuation to our on-going studies toward the development of new routes for the synthesis of heterocyclic compounds [19-22], we herein report a simple, convenient, and environmentally benign method for the synthesis of 13-arylindeno[1,2- $b]$ naphtha[1,2-e]pyran-12(13H)-one derivatives via the one step three-component coupling of an aromatic aldehyde with $\beta$-naphthol and $2 H$-indene-1,3-dione in the presence of a $\mathrm{P}(4-\mathrm{VPH}) \mathrm{HSO}_{4}$ catalyst under solvent-free conditions at 80 ${ }^{\circ} \mathrm{C}$ (Scheme 1).

\section{Experimental}

\subsection{Apparatus and analysis}

All of the chemicals used were purchased from Merck, Fluka, and Aldrich Chemical Companies. All of the yields reported in this study refer to isolated products unless otherwise stated. ${ }^{1} \mathrm{H}$ NMR (500 MHz) and ${ }^{13} \mathrm{C}$ NMR (125 MHz) spectra were obtained using a Bruker Avance DRX-500 at ambient temperature using TMS as internal reference. FT-IR spectra were obtained as $\mathrm{KBr}$ discs on a Shimadzu spectrometer. Mass spectra were determined on a Varion Saturn 2000 GC/MS instrument. Elemental analyses were measured on a Perkin Elmer $2400 \mathrm{CHN}$ elemental analyzer flowchart.

\subsection{Preparation of poly(4-vinylpyridinium)hydrogen sulfate}

Poly(4-vinylpyridinium)hydrogen sulfate was prepared as reported in the literature [15]. $\mathrm{H}_{2} \mathrm{SO}_{4}(0.3 \mathrm{~mL}, 5.3 \mathrm{mmol}$, as a $96 \%$ standard solution) was added to a suspension of powdered poly(4-vinyl pyridine) $(1.0 \mathrm{~g})$ in dry methanol $(10 \mathrm{~mL})$, and the resulting mixture was stirred at room temperature for $8 \mathrm{~h}$. The methanol was then removed under reduced pressure to give the $\mathrm{P}(4-\mathrm{VPH}) \mathrm{HSO}_{4}$ catalyst. The residual sulfuric acid was washed out with deionized water until no sulfate anions could be detected in the liquid (as determined by their reaction with $\mathrm{BaCl}_{2}$ ), and the resulting solid powder was dried under vacuum at $65{ }^{\circ} \mathrm{C}$ for $48 \mathrm{~h}$ to afford $\mathrm{P}(4-\mathrm{VPH}) \mathrm{HSO}_{4}(0.5 \mathrm{mmol} / \mathrm{g})$ as a pale yellow powder ( $1.3 \mathrm{~g}, 85 \%$ yield). The catalyst was washed with solvent and dried before being reused for subsequent reactions.

\subsection{Preparation of 13-aryl-indeno[1,2-b]naphtha[1,2-e] pyran-12(13H)-one}

$\mathrm{P}(4-\mathrm{VPH}) \mathrm{HSO}_{4}$ (15 mg, $0.0075 \mathrm{mmol}$ ) was added to a mixture of aldehyde ( $1 \mathrm{mmol}), \beta$-naphthol $(1 \mathrm{mmol})$, and $2 \mathrm{H}$-indene-1,3-dione ( $1 \mathrm{mmol})$, and the resulting mixture was stirred at $80{ }^{\circ} \mathrm{C}$ for an appropriate time at atmospheric pressure. Upon completion of the reaction (as determined by TLC), the mixture was diluted with $\mathrm{CH}_{2} \mathrm{Cl}_{2}(20 \mathrm{~mL})$, and the solid catalyst was removed by filtration. The filtrate was then collected and evaporated to dryness to give the crude product, which was purified by silica gel column chromatography using $\mathrm{CH}_{2} \mathrm{Cl}_{2}$ as an eluent.

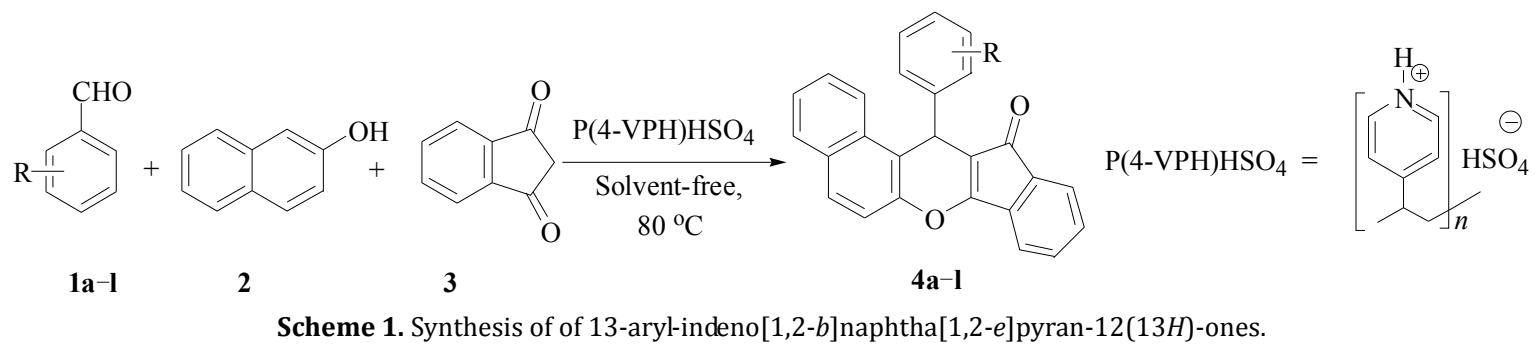




\subsection{Spectral data for the synthesized compounds (4a-I)}

13-Phenyl-indeno[1,2-b]naphtho[1,2-e]pyran-12(13H)-one (4a). Yellow solid. IR (KBr, $\left.\mathrm{cm}^{-1}\right)$ : 2970, 1645, 1592, 1370, 1217, 1188; ${ }^{1} \mathrm{H}$ NMR (500 MHz, $\mathrm{CDCl}_{3}$ ): $\delta 5.55$ (s, 1H, CH), 7.11 (t, $J=7.4 \mathrm{~Hz}, 1 \mathrm{H}, \mathrm{Ar}-\mathrm{H}), 7.22(\mathrm{t}, J=7.6 \mathrm{~Hz}, 2 \mathrm{H}, \mathrm{Ar}-\mathrm{H}), 7.29-7.44$ (m, 8H, Ar-H), 7.54 (d, J = 8.8 Hz, 1H, Ar-H), 7.82-7.90 (m, 3H, $\mathrm{Ar}-\mathrm{H}) ;{ }^{13} \mathrm{C}$ NMR $\left(125 \mathrm{MHz}, \mathrm{CDCl}_{3}\right): \delta 33.9,111.2,115.8,116.9$, $117.9,120.8,123.2,124.7,125.6,126.3,127.7,128.0,129.1$, 130.6, 131.4, 131.8, 132.0, 132.5, 137.2, 142.8, 148.9, 166.9, 193.5; MS (ESI): $m / z 361[\mathrm{M}+\mathrm{H}]^{+}$; Anal. Calcd for $\mathrm{C}_{26} \mathrm{H}_{16} \mathrm{O}_{2}: \mathrm{C}$ 86.66\%, H 4.44\%; Found: C 86.58\%, H 4.46\%.

13-(4-Fluorophenyl)-indeno[1,2-b]naphtho[1,2-e]pyran-12 (13H)-one (4b). Yellow solid. IR (KBr, $\left.\mathrm{cm}^{-1}\right)$ : 2966, 1659, 1588, 1369, 1216, 1194; ${ }^{1} \mathrm{H}$ NMR (500 MHz, $\left.\mathrm{CDCl}_{3}\right): \delta 5.54(\mathrm{~s}, 1 \mathrm{H}, \mathrm{CH})$, $7.11(\mathrm{~d}, J=8.4 \mathrm{~Hz}, 2 \mathrm{H}, \mathrm{Ar}-\mathrm{H}), 7.22-7.41$ (m, 8H, Ar-H), 7.56 (d, $J$ $=8.6 \mathrm{~Hz}, 1 \mathrm{H}, \mathrm{Ar}-\mathrm{H}), 7.69(\mathrm{t}, J=9.1 \mathrm{~Hz}, 1 \mathrm{H}, \mathrm{Ar}-\mathrm{H}), 7.78-7.85(\mathrm{~m}$, $2 \mathrm{H}, \mathrm{Ar}-\mathrm{H}) ;{ }^{13} \mathrm{C}$ NMR $\left(125 \mathrm{MHz}, \mathrm{CDCl}_{3}\right): \delta 34.5,111.7,116.2$, $116.7,117.8,120.7,123.7,125.0,126.0,127.4,128.1,128.8$, 129.5, 130.0, 130.9, 131.5, 132.6, 133.1, 137.5, 143.3, 149.4, 166.3, 192.7; MS (ESI): $m / z 379[\mathrm{M}+\mathrm{H}]^{+}$; Anal. Calcd for $\mathrm{C}_{26} \mathrm{H}_{15} \mathrm{FO}_{2}$ : C 82.54\%, H 3.97\%; Found: C 82.44\%, H 3.93\%.

13-(4-Methylphenyl)-indeno[1,2-b]naphtho[1,2-e]pyran-12 (13H)-one (4c). Yellow solid. IR (KBr, $\left.\mathrm{cm}^{-1}\right)$ : 2956, 1653, 1584, 1365, 1224, 1180; ${ }^{1} \mathrm{H}$ NMR (500 MHz, $\mathrm{CDCl}_{3}$ ): $\delta 2.28(\mathrm{~s}, 3 \mathrm{H}$, $\mathrm{CH}_{3}$ ), 5.60 (s, 1H, CH), 7.06 (d, J = 8.4 Hz, 2H, Ar-H), 7.24-7.43 (m, 8H, Ar-H), 7.54 (d, $J=8.8 \mathrm{~Hz}, 1 \mathrm{H}, \mathrm{Ar}-\mathrm{H}), 7.70(\mathrm{t}, J=9.0 \mathrm{~Hz}$, 1H, Ar-H), 7.81-7.87 (m, 2H, Ar-H); ${ }^{13} \mathrm{C}$ NMR (125 MHz, $\left.\mathrm{CDCl}_{3}\right)$ : $\delta 18.3,34.8,111.6,115.5,117.3,118.3,121.2,123.8,125.3$, $126.4,127.2,127.9,128.4,129.3,129.9,130.7,131.3,132.2$, 132.9, 136.5, 142.7, 148.6, 167.0, 192.6; MS (ESI): $m / z 375$ $[\mathrm{M}+\mathrm{H}]^{+}$; Anal. Calcd for $\mathrm{C}_{27} \mathrm{H}_{18} \mathrm{O}_{2}$ : C 86.63\%, H 4.81\%; Found: C 86.69\%, H $4.80 \%$.

13-(4-Methoxyphenyl)-indeno[1,2-b]naphtho[1,2-e]pyran12(13H)-one (4d). Yellow solid. IR (KBr, $\left.\mathrm{cm}^{-1}\right)$ : 2955, 1647, 1591, 1375, 1220, 1190; ${ }^{1} \mathrm{H}$ NMR (500 MHz, $\mathrm{CDCl}_{3}$ ): $\delta 3.58$ (s, $\left.3 \mathrm{H}, \mathrm{OCH}_{3}\right), 5.56(\mathrm{~s}, 1 \mathrm{H}, \mathrm{CH}), 7.10(\mathrm{~d}, J=8.4 \mathrm{~Hz}, 2 \mathrm{H}, \mathrm{Ar}-\mathrm{H})$, 7.19-7.39 (m, 8H, Ar-H), 7.49 (d, $J=8.4 \mathrm{~Hz}, 1 \mathrm{H}, \mathrm{Ar}-\mathrm{H}), 7.68$ (t, $J$ $=9.0 \mathrm{~Hz}, 1 \mathrm{H}, \mathrm{Ar}-\mathrm{H}), 7.79-7.87(\mathrm{~m}, 2 \mathrm{H}, \mathrm{Ar}-\mathrm{H}) ;{ }^{13} \mathrm{C}$ NMR $(125$ $\left.\mathrm{MHz}, \mathrm{CDCl}_{3}\right): \delta: 33.8,51.3,110.8,116.1,117.0,118.4,121.0$, $123.3,125.5,126.1,126.7,128.0,128.6,129.4,130.3,131.0$, 131.8, 132.8, 133.3, 136.8, 143.4, 148.8, 167.2, 193.0; MS (ESI): $\mathrm{m} / \mathrm{z} 391[\mathrm{M}+\mathrm{H}]^{+}$; Anal. Calcd for $\mathrm{C}_{27} \mathrm{H}_{18} \mathrm{O}_{3}$ : C 83.08\%, H 4.61\%; Found: C 83.01\%, H 4.64\%.

13-(3-Nitrophenyl)-indeno[1,2- $b]$ naphtho[1,2-e]pyran-12 (13H)-one (4e). Yellow solid. IR (KBr, $\left.\mathrm{cm}^{-1}\right)$ : 2952, 1662, 1587, 1371, 1227, 1177 ; ${ }^{1} \mathrm{H}$ NMR (500 MHz, $\left.\mathrm{CDCl}_{3}\right): \delta 5.70$ (s, $1 \mathrm{H}, \mathrm{CH}$ ), 7.28-7.34 (m, 1H, Ar-H), 7.42-7.49 (m, 6H, Ar-H), 7.59 (d, $J=$ $9.2 \mathrm{~Hz}, 1 \mathrm{H}, \mathrm{Ar}-\mathrm{H}$ ), 7.65 (d, $J=8.8 \mathrm{~Hz}, 1 \mathrm{H}, \mathrm{Ar}-\mathrm{H}), 7.78$ (d, $J=7.8$ $\mathrm{Hz}, 1 \mathrm{H}, \mathrm{Ar}-\mathrm{H}$ ), 7.85-7.88 (m, 1H, Ar-H), 7.92 (d, J = 8.8 Hz, 1H, $\mathrm{Ar}-\mathrm{H}$ ), 7.97 (d, J = 8.6 Hz, 1H, Ar-H), 8.02 (s, 1H, Ar-H); ${ }^{13} \mathrm{C}$ NMR $\left(125 \mathrm{MHz}, \mathrm{CDCl}_{3}\right): \delta 35.0,110.4,115.3,116.6,117.7,121.4$, $124.1,124.6,125.7,126.5,127.9,128.2,128.8,130.5,131.5$, $131.9,132.5,133.4,137.3,143.6,148.6,167.6,193.4$; MS (ESI): $\mathrm{m} / \mathrm{z} 406$ [M+H] ${ }^{+}$; Anal. Calcd for $\mathrm{C}_{26} \mathrm{H}_{15} \mathrm{NO}_{4}: \mathrm{C} 77.04 \%$, H 3.70\%, N 3.46\%; Found: C 77.07\%, H 3.60\%, N 3.36\%.

13-(4-Chlorophenyl)-indeno[1,2-b]naphtho[1,2-e]pyran-12
(13H)-one (4f). Yellow solid. IR (KBr, $\left.\mathrm{cm}^{-1}\right)$ : 2962, 1664, 1594, $1363,1219,1186$; ${ }^{1} \mathrm{H}$ NMR $\left(500 \mathrm{MHz}, \mathrm{CDCl}_{3}\right): \delta 5.69$ (s, $\left.1 \mathrm{H}, \mathrm{CH}\right)$, 7.05 (d, $J=8.4 \mathrm{~Hz}, 2 \mathrm{H}, \mathrm{Ar}-\mathrm{H}$ ), 7.20-7.41 (m, 8H, Ar-H), 7.58 (d, $J$ $=8.8 \mathrm{~Hz}, 1 \mathrm{H}, \mathrm{Ar}-\mathrm{H}), 7.73(\mathrm{t}, J=9.2 \mathrm{~Hz}, 1 \mathrm{H}, \mathrm{Ar}-\mathrm{H}), 7.82-7.90(\mathrm{~m}$, $2 \mathrm{H}, \mathrm{Ar}-\mathrm{H}) ;{ }^{13} \mathrm{C}$ NMR $\left(125 \mathrm{MHz}, \mathrm{CDCl}_{3}\right): \delta 35.3,110.5,116.4$, 117.1, 118.0, 120.5, 123.9, 124.8, 125.9, 127.0, 128.1, 128.7, 129.2, 129.8, 130.6, 131.4, 131.9, 132.5, 136.5, 142.9, 149.1, 166.8, 192.7; MS(ESI): $m / z \quad 395[\mathrm{M}+\mathrm{H}]^{+}$; Anal. Calcd for $\mathrm{C}_{26} \mathrm{H}_{15} \mathrm{ClO}_{2}$ : C 79.10\%, H 3.80\%; Found: C 79.01\%, H 3.83\%.

13-(2-Chlorophenyl)-indeno[1,2-b]naphtho[1,2-e]pyran-12 (13H)-one (4g). Yellow solid. IR (KBr, cm-1): 2973, 1647, 1590, $1362,1216,1184 ;{ }^{1} \mathrm{H}$ NMR $\left(500 \mathrm{MHz}, \mathrm{CDCl}_{3}\right): \delta 5.82(\mathrm{~s}, 1 \mathrm{H}, \mathrm{CH})$, 7.02-7.10 (m, 3H, Ar-H), 7.25-7.50 (m, 8H, Ar-H), 7.82-7.90 (m, $3 \mathrm{H}, \mathrm{Ar}-\mathrm{H}) ;{ }^{13} \mathrm{C}$ NMR $\left(125 \mathrm{MHz}, \mathrm{CDCl}_{3}\right): \delta 35.4,111.0,115.7$, 116.7, 117.7, 120.8, 124.4, 124.9, 126.0, 127.2, 127.9, 128.6, 129.1, 130.2, 131.3, 131.8, 132.6, 132.9, 136.8, 143.7, 149.4, 168.9, 192.9; MS (ESI): $m / z 395[\mathrm{M}+\mathrm{H}]^{+}$; Anal. Calcd for $\mathrm{C}_{26} \mathrm{H}_{15} \mathrm{ClO}_{2}$ : C 79.10\%, H 3.80\%; Found: C 79.12\%, H 3.72\%.

13-(4-Bromophenyl)-indeno[1,2-b]naphtho[1,2-e]pyran-12 (13H)-one (4h). Yellow solid. IR (KBr, cm-1): 2964, 1659, 1585, 1372, 1222, 1192; ${ }^{1} \mathrm{H}$ NMR (500 MHz, $\left.\mathrm{CDCl}_{3}\right): \delta 5.49$ (s, $\left.1 \mathrm{H}, \mathrm{CH}\right)$, 7.15 (d, $J=8.4 \mathrm{~Hz}, 2 \mathrm{H}, \mathrm{Ar}-\mathrm{H}$ ), 7.28-7.46 (m, 8H, Ar-H), 7.55 (d, $J$ $=8.8 \mathrm{~Hz}, 1 \mathrm{H}, \mathrm{Ar}-\mathrm{H}), 7.65(\mathrm{t}, J=9.0 \mathrm{~Hz}, 1 \mathrm{H}, \mathrm{Ar}-\mathrm{H}), 7.77-7.85(\mathrm{~m}$, $2 \mathrm{H}, \mathrm{Ar}-\mathrm{H}) ;{ }^{13} \mathrm{C}$ NMR $\left(125 \mathrm{MHz}, \mathrm{CDCl}_{3}\right): \delta 34.7,110.6,115.9$, 117.5, 118.0, 121.3, 124.0, 125.4, 126.4, 127.3, 128.3, 128.7, 129.1, 130.1, 130.9, 131.5, 132.2, 132.7, 137.6, 143.0149 .5 , 167.2, 192.5; MS (ESI): $m / z 439.5[\mathrm{M}+\mathrm{H}]^{+}$; Anal. Calcd for $\mathrm{C}_{26} \mathrm{H}_{15} \mathrm{BrO}_{2}$ : C 71.09\%, H 3.42\%; Found: C 71.04\%, H 3.49\%.

13-(4-Nitrophenyl)-indeno[1,2-b]naphtho[1,2-e]pyran-12 (13H)-one (4i). Yellow solid. IR (KBr, $\left.\mathrm{cm}^{-1}\right): 2958,1655,1597$, $1377,1220,1193 ;{ }^{1} \mathrm{H}$ NMR (500 MHz, $\left.\mathrm{CDCl}_{3}\right): \delta 5.57(\mathrm{~s}, 1 \mathrm{H}, \mathrm{CH})$, 7.13 (d, $J=8.4 \mathrm{~Hz}, 2 \mathrm{H}, \mathrm{Ar}-\mathrm{H}), 7.24-7.41(\mathrm{~m}, 8 \mathrm{H}, \mathrm{Ar}-\mathrm{H}), 7.50$ (d, $J$ $=8.8 \mathrm{~Hz}, 1 \mathrm{H}, \mathrm{Ar}-\mathrm{H}), 7.71(\mathrm{t}, J=9.2 \mathrm{~Hz}, 1 \mathrm{H}, \mathrm{Ar}-\mathrm{H}), 7.84-7.87(\mathrm{~m}$, $2 \mathrm{H}, \mathrm{Ar}-\mathrm{H})$; ${ }^{13} \mathrm{C}$ NMR $\left(125 \mathrm{MHz}, \mathrm{CDCl}_{3}\right): \delta 34.0,110.7,116.0$, $117.2,118.1,121.1,123.9,125.0,126.3,126.9,127.7,128.2$, 129.0, 129.7, 131.1, 131.8, 132.5, 133.2, 136.6, 143.2, 148.7, 167.1, 193.7; MS (ESI): $m / z 406[\mathrm{M}+\mathrm{H}]^{+}$; Anal. Calcd for $\mathrm{C}_{26} \mathrm{H}_{15} \mathrm{NO}_{4}$ : C 77.04\%, H 3.70\%, N 3.46\%; Found: C 73.94\%, H $3.75 \%$, N 3.37\%.

13-(2-Fluorophenyl)-indeno[1,2-b]naphtho[1,2-e]pyran-12 (13H)-one (4j). Yellow solid. IR (KBr, $\left.\mathrm{cm}^{-1}\right): 2971,1657,1583$, 1372, 1218, 1178; ${ }^{1} \mathrm{H}$ NMR (500 MHz, $\left.\mathrm{CDCl}_{3}\right): \delta 5.76(\mathrm{~s}, 1 \mathrm{H}, \mathrm{CH})$, 7.06-7.12 (m, 3H, Ar-H), 7.21-7.48 (m, 8H, Ar-H), 7.79-7.88 (m, $3 \mathrm{H}, \mathrm{Ar}-\mathrm{H}) ;{ }^{13} \mathrm{C}$ NMR $\left(125 \mathrm{MHz}, \mathrm{CDCl}_{3}\right): \delta 34.8,111.1,115.7$, $116.9,118.3,120.5,124.3,125.1,126.2,127.4,128.0,128.6$, 129.2, 130.4, 131.6, 131.9, 132.4, 132.8, 136.8, 143.1, 149.0, 166.6, 192.8; MS (ESI): $m / z 379[\mathrm{M}+\mathrm{H}]^{+}$; Anal. Calcd for $\mathrm{C}_{26} \mathrm{H}_{15} \mathrm{FO}_{2}$ : C 82.54\%, H 3.97\%; Found: C 82.57\%, H 3.87\%.

13-(2,4-Dichlorophenyl)-indeno[1,2-b]naphtho[1,2-e]pyran -12(13H)-one (4k). Yellow solid. IR (KBr, cm-1): 2959, 1653, 1593, 1369, 1223, 1180; ${ }^{1} \mathrm{H}$ NMR (500 MHz, $\left.\mathrm{CDCl}_{3}\right): \delta 5.77$ (s, $1 \mathrm{H}, \mathrm{CH}), 7.00-7.08(\mathrm{~m}, 2 \mathrm{H}, \mathrm{Ar}-\mathrm{H}), 7.30-7.35(\mathrm{~m}, 1 \mathrm{H}, \mathrm{Ar}-\mathrm{H})$, 7.44-7.53 (m, 7H, Ar-H), 7.80-7.88 (m, 3H, Ar-H); ${ }^{13} \mathrm{C}$ NMR (125 MHz, $\left.\mathrm{CDCl}_{3}\right): \delta 35.2,110.7,115.5,117.2,118.4,121.3$, $124.4,124.9,125.8,126.4,127.7,128.3,129.1,129.9,130.7$, $131.5,131.9,132.4,137.0,142.7,149.3,167.5,193.6$; MS (ESI): $\mathrm{m} / \mathrm{z} 429.5[\mathrm{M}+\mathrm{H}]^{+}$; Anal. Calcd for $\mathrm{C}_{26} \mathrm{H}_{14} \mathrm{Cl}_{2} \mathrm{O}_{2}$ : C $72.74 \%, \mathrm{H}$ 
3.26\%; Found: C 72.66\%, H 3.23\%.

13-(3,4-Dichlorophenyl)-indeno[1,2- $b]$ naphtho[1,2-e]pyran -12(13H)-one (4l). Yellow solid. IR ( $\left.\mathrm{KBr}, \mathrm{cm}^{-1}\right): 2972,1661$, 1579, 1365, 1225, 1187; ${ }^{1} \mathrm{H}$ NMR (500 MHz, $\mathrm{CDCl}_{3}$ ): $\delta 5.88$ (s, $1 \mathrm{H}, \mathrm{CH}), 6.98-7.05(\mathrm{~m}, 2 \mathrm{H}, \mathrm{Ar}-\mathrm{H}), 7.34-7.39(\mathrm{~m}, 1 \mathrm{H}, \mathrm{Ar}-\mathrm{H})$, 7.46-7.54 (m, 7H, Ar-H), 7.77-7.85 (m, 3H, Ar-H); ${ }^{13} \mathrm{C}$ NMR $\left(125 \mathrm{MHz}, \mathrm{CDCl}_{3}\right): \delta 35.1,111.3,116.2,117.6,118.0,121.0$, $123.3,125.3,126.5,127.2,128.3,128.8,129.5,130.6,131.3$, 131.6, 132.1, 132.7, 136.9, 142.8, 148.7, 166.3, 193.1; MS (ESI): $\mathrm{m} / \mathrm{z} 429.7[\mathrm{M}+\mathrm{H}]^{+}$; Anal. Calcd for $\mathrm{C}_{26} \mathrm{H}_{14} \mathrm{Cl}_{2} \mathrm{O}_{2}$ : C $72.74 \%, \mathrm{H}$ 3.26\%; Found: C 72.71\%, H 3.29\%.

\section{Results and discussion}

As part of our initial study to determine the optimum reaction conditions for this transformation, the reaction of benzaldehyde $(1 \mathrm{mmol})$ with $\beta$-naphthol $(1 \mathrm{mmol})$ and $2 H$-indene1,3-dione (1 mmol) was examined as a model reaction in the presence of $15 \mathrm{mg}$ of $\mathrm{P}(4-\mathrm{VPH}) \mathrm{HSO}_{4}$ as a catalyst under refluxing conditions in a variety of different solvents, including $\mathrm{CH}_{3} \mathrm{CN}$, 1,4-dioxane, EtOH, DMF, and $\mathrm{CHCl}_{3}$ (Table 1, entries 1-5). The reaction was also conducted under solvent-free conditions at $80{ }^{\circ} \mathrm{C}$, where it proceeded to completion within 30 min (Table 1, entry 6). The reaction was also investigated at a variety of different temperatures, including room temperature, as well as $50,60,70,80$, and $90^{\circ} \mathrm{C}$ (Table 1 , entries 6-11). The greatest yield with the shortest reaction time was obtained under the solvent-free conditions at $80^{\circ} \mathrm{C}$.

The optimal amount of the catalyst was determined by conducting a series of reactions involving a 0-, 5-, 10-, 15-, 20-, or 25-mg charge of the $\mathrm{P}(4-\mathrm{VPH}) \mathrm{HSO}_{4}$ catalyst under solvent-free conditions at $80{ }^{\circ} \mathrm{C}$ (Table 1 , entries 6 and 12-16). The results of these experiments demonstrated that a 15-mg charge of the $\mathrm{P}(4-\mathrm{VPH}) \mathrm{HSO}_{4}$ catalyst provided the highest yield of the product at $80{ }^{\circ} \mathrm{C}$ (Table 1, entry 6). The use of a smaller amount of catalyst resulted in a lower yield, whereas the use of a larger amount did not have any impact on the reaction time or the yield (Table 1, entries 6 and 12-16). A blank reaction was carried out involving benzaldehyde, $\beta$-naphthol, and $2 \mathrm{H}$-indene-1,3-dione in the absence of the catalyst under the optimized conditions to determine the role of the catalyst. This reaction did not provide any of the desired product and confirmed the importance of the catalyst to the reaction (Table 1, entry 12).

To evaluate the scope of this catalytic transformation, the optimized reaction conditions were subsequently applied to the reaction of $2 \mathrm{H}$-indene-1,3-dione with a variety of different aromatic aldehydes and $\beta$-naphthol (Table 2, entries 1-12). A wide range of aromatic aldehydes bearing either electron-donating or electron-withdrawing substituents reacted successfully with $2 H$-indene-1,3-dione and $\beta$-naphthol under the optimized conditions to give the corresponding 13-aryl-indeno[1,2$b]$ naphtha[1,2-e]pyran-12(13H)-one derivatives in high yields over short reaction time.

Based on these results, it was clear that the electronic and steric effects of the substituents on the aldehydes had a significant impact on the yields of the products. In comparison with electron-donating groups, such as the methyl and methoxy groups (Table 2, entries 3 and 4), electron-withdrawing groups, as well as aromatic aldehydes substituted with halogen atoms, gave shorter reaction time (Table 2, entries 5, 6, 8, and 9). When ortho substituted aldehydes were used, they gave the corresponding products in good yields but required a longer reaction time (Table 2, entry 7). In contrast to aromatic aldehydes, aliphatic aldehydes could not be used under the optimized conditions and failed to provide any of the desired products.

In the interests of green chemistry and developing an environmentally benign process, we decided to study the catalytic activity of recycled solid acid $\mathrm{P}(4-\mathrm{VPH}) \mathrm{HSO}_{4}$ for the synthesis of 13-aryl-indeno[1,2-b]naphtha[1,2-e]pyran-12(13H)-one using the model reaction system under the optimized conditions (Fig.

Table 1

Synthesis of 13 -aryl-indeno[1,2-b]naphtha[1,2-e]pyran-12(13H)-one in the presence of the $\mathrm{P}(4-\mathrm{VPH}) \mathrm{HSO}_{4}$ catalyst under different reaction conditions.

\begin{tabular}{|c|c|c|c|c|c|}
\hline Entry & Solvent & Catalyst (mg) & Temperature $\left({ }^{\circ} \mathrm{C}\right)$ & Time (min) & Yield a $(\%)$ \\
\hline 1 & $\mathrm{CH}_{3} \mathrm{CN}$ & 15 & reflux & 120 & 36 \\
\hline 2 & 1,4-dioxane & 15 & reflux & 120 & 44 \\
\hline 3 & $\mathrm{EtOH}$ & 15 & reflux & 120 & 69 \\
\hline 4 & DMF & 15 & reflux & 120 & 43 \\
\hline 5 & $\mathrm{CHCl}_{3}$ & 15 & reflux & 120 & 42 \\
\hline 6 & solvent-free & 15 & 80 & 40 & 92 \\
\hline 7 & solvent-free & 15 & RT & 120 & 41 \\
\hline 8 & solvent-free & 15 & 50 & 120 & 56 \\
\hline 9 & solvent-free & 15 & 60 & 90 & 68 \\
\hline 10 & solvent-free & 15 & 70 & 60 & 81 \\
\hline 11 & solvent-free & 15 & 90 & 40 & 92 \\
\hline 12 & solvent-free & 0 & 80 & 90 & - \\
\hline 13 & solvent-free & 5 & 80 & 40 & 44 \\
\hline 14 & solvent-free & 10 & 80 & 40 & 72 \\
\hline 15 & solvent-free & 20 & 80 & 40 & 92 \\
\hline 16 & solvent-free & 25 & 80 & 40 & 93 \\
\hline
\end{tabular}

Reaction conditions: benzaldehyde (1 mmol), $\beta$-naphthol (1 mmol), $2 \mathrm{H}$-indene-1,3-dione (1 mmol). ${ }^{\text {Is }}$ - 1 ated yield. 
Table 2

Synthesis of various 13-aryl-indeno[1,2-b]naphtha[1,2-e]pyran-12(13H)-ones.

\begin{tabular}{|c|c|c|c|c|c|c|c|c|c|c|c|}
\hline Entry & Aldehyde & Product & $\begin{array}{l}\text { Time } \\
\text { (min) }\end{array}$ & $\begin{array}{c}\text { Yield a } \\
(\%)\end{array}$ & $\begin{array}{c}\text { Melting } \\
\text { point }\left({ }^{\circ} \mathrm{C}\right)\end{array}$ & Entry & Aldehyde & Product & $\begin{array}{l}\text { Time } \\
\text { (min) }\end{array}$ & $\begin{array}{c}\text { Yield a }^{\text {a }} \\
(\%)\end{array}$ & $\begin{array}{c}\text { Melting } \\
\text { point }\left({ }^{\circ} \mathrm{C}\right)\end{array}$ \\
\hline 1 & & & 40 & 92 & $\begin{array}{c}202-204 \\
(202-203 \\
[13])\end{array}$ & 7 & & & 60 & 86 & $\begin{array}{c}240-241 \\
(240-241 \\
[13])\end{array}$ \\
\hline 2 & & & 30 & 93 & $\begin{array}{c}206-208 \\
(208-209 \\
[13])\end{array}$ & 8 & & & 30 & 93 & $208-210$ \\
\hline 3 & $\mathrm{H}_{3}$ & & 60 & 89 & $\begin{array}{c}193-195 \\
(192-193 \\
[13])\end{array}$ & 9 & & & 30 & 92 & $220-222$ \\
\hline 4 & $\mathrm{CH}_{3}$ & & 60 & 90 & $\begin{array}{c}224-226 \\
(225-226 \\
[13])\end{array}$ & 10 & & & 60 & 87 & $228-230$ \\
\hline 5 & & & 40 & 90 & $\begin{array}{c}242-243 \\
(240-241 \\
[13])\end{array}$ & 11 & & & 30 & 92 & $\begin{array}{c}250-252 \\
(252-253 \\
[13])\end{array}$ \\
\hline 6 & $\mathrm{Cl}$ & & 30 & 92 & $\begin{array}{c}224-226 \\
(225-226 \\
[13])\end{array}$ & 12 & & & 30 & 91 & $\begin{array}{c}244-245 \\
(245-246 \\
[13])\end{array}$ \\
\hline
\end{tabular}

1). After the separation of product, the catalyst was washed with $\mathrm{CH}_{2} \mathrm{Cl}_{2}$ and vacuum dried to remove any residual $\mathrm{CH}_{2} \mathrm{Cl}_{2}$, and the resulting catalyst was reused directly in the next run.

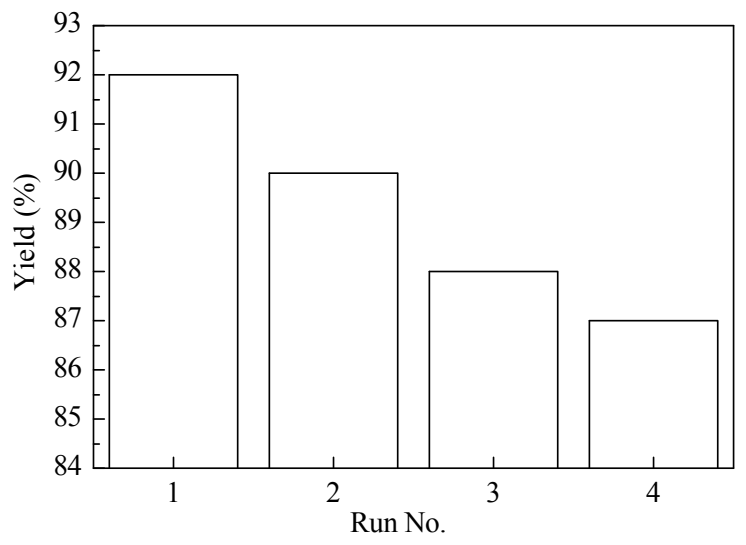

Fig. 1. Reusability of $\mathrm{P}(4-\mathrm{VPH}) \mathrm{HSO}_{4}$.
As shown in Fig. 1, the solid acid $\mathrm{P}(4-\mathrm{VPH}) \mathrm{HSO}_{4}$ could be recycled at least four times without any significant reduction in its catalytic activity, with yields in the range of $87 \%$ to $92 \%$.

A probable mechanism for the formation of the 13-aryl-indeno[1,2-b]naphtha[1,2-e]pyran-12(13H)-ones using $\mathrm{P}(4-\mathrm{VPH}) \mathrm{HSO}_{4}$ as a catalyst is shown in Scheme 2 . The protonated form of the aldehyde would be formed under the acidic conditions, as well as the enol form of the diketone (intermediate $\mathbf{c}$ ), which would exist in equilibrium. Intermediate a would then be formed from the nucleophilic attack of 2-naththol on the protonated aldehyde. Subsequent protonation of intermediate a followed by the removal of water would result in the formation of intermediate $\mathbf{b}$, which has been well documented in the literature of ortho-quinone methides (o-QMs) [23]. The nucleophilic attach of intermediate $\mathbf{c}$ on the protonated form of intermediate b would give the acyclic adduct intermediate, which would undergo intramolecular cyclization, followed by the loss of water to form the targeted molecules. 


\section{Graphical Abstract}

Chin. J. Catal., 2014, 35: 43-48 doi: 10.1016/S1872-2067(12)60707-4

Poly(4-vinylpyridinium)hydrogen sulfate: A novel and efficient catalyst for the synthesis of 13-aryl-indeno[1,2-b]naphtha[1,2-e]pyran$12(13 H)$-ones under solvent-free conditions

Majid Ghashang, Syed Sheik Mansoor*, Krishnamoorthy Aswin Najafabad Branch, Islamic Azad University, Iran; C. Abdul Hakeem College, India

A simple and efficient method for the synthesis of 13-aryl-indeno[1,2- $b$ ] naphtha[1,2-e]pyran-12(13H)-ones has been developed that proceeds via a one-pot three-component sequential reaction involving an aromatic aldehyde, $\beta$-naphthol, and $2 H$-indene-1,3-dione under solvent-free conditions catalyzed by poly(4-vinylpyridinium)hydrogen sulfate $\left(\mathrm{P}(4-\mathrm{VPH}) \mathrm{HSO}_{4}\right)$.

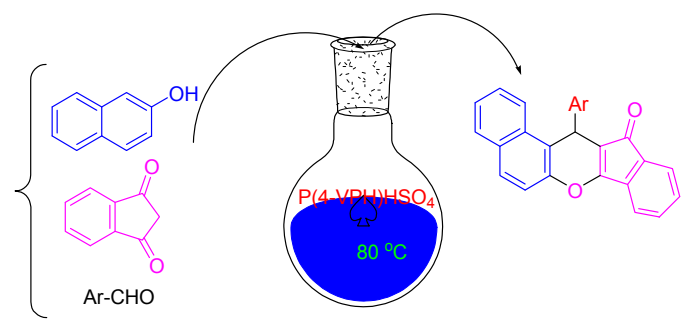

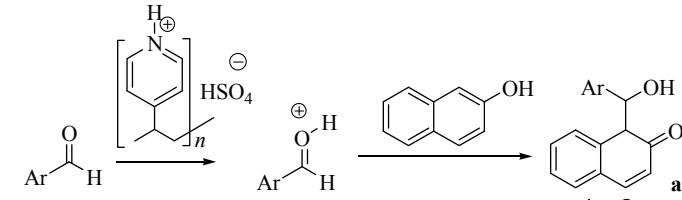<smiles>O=C1CC(=O)c2ccccc2C1=Cc1ccccc1</smiles>

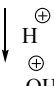<smiles>C/C=C\C(C=O)C(O)[AlH2]</smiles><smiles></smiles>
$-\mathrm{H}_{2} \mathrm{O}$

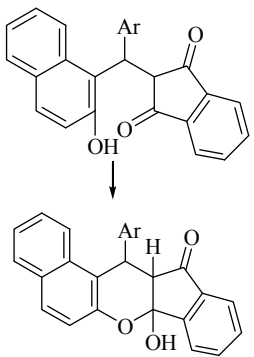

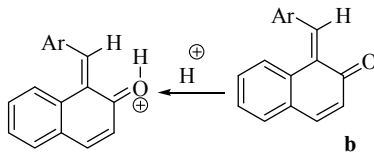
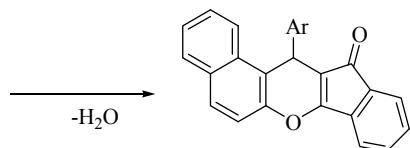

Scheme 2. Proposed mechanism for the formation of 13-aryl-indeno[1,2-b]naphtha[1,2-e]pyran-12(13H)-ones.

\section{Conclusions}

A simple and highly efficient method for the synthesis of 13-aryl-indeno[1,2- $b]$ naphtha[1,2-e]pyran-12(13H)-ones has been developed via the $\mathrm{P}(4-\mathrm{VPH}) \mathrm{HSO}_{4}$ catalyzed condensation of an aromatic aldehyde with $\beta$-naphthol and $2 \mathrm{H}$-indene-1,3dione under solvent-free conditions at $80{ }^{\circ} \mathrm{C}$. The current approach offers the advantages of simple methodology, clean and mild reaction conditions, high atom-economy, short reaction time, low environmental impact, wide substrate scope, high yields, and excellent product purity.

\section{Acknowledgments}

The author Mansoor would like to express his gratitude to the management of C. Abdul Hakeem College, Melvisharam, India, for the use of their facilities and their general support.

\section{References}

[1] Dömling A, Ugi I. Angew Chem Int Ed, 2000, 39: 3168

[2] Schreiber S L. Science, 2000, 287: 1964

[3] Davoodnia A, Khashi M, Tavakoli-Hoseini N. Chin J Catal (催化学 报), 2013, 34: 1173

[4] Martinez-Grau A, Marco J L. Bioorg Med Chem Lett, 1997, 7: 3165

[5] Hiramoto K, Nasuhara A, Michikoshi K, Kato T, Kikugawa K. Mutation Res, 1997, 395: 47

[6] Khafagy M M, Abd El-Wahab A H F, Eid F A, El-Agrody A M. Fármaco, 2002, 57: 715

[7] Dell C P, Smith C W. EP Patent 537949. 1993

[8] Anderson D R, Hegde S, Reinhard E, Gomez L, Vernier W F, Lee L, Liu S, Sambandam A, Snider P A, Masih L. Bioorg Med Chem Lett, 2005, 15: 1587

[9] Skommer J, Wlodkowic D, Mättö M, Eray M, Pelkonen J. Leukem Res, 2006, 30: 322

[10] Pelz K, Dobson T A. US Patent 3904 617. 1975

[11] Goerlitzer K, Dehne A, Engler E. Arch Pharm, 1983, 316: 264

[12] Jirkovsky I, Humber L G, Noureldin R. Eur J Med Chem, 1976, 11 : 571

[13] Wu L Q, Yang L M, Wang X, Yan F L. J Chin Chem Soc, 2010, 57: 738

[14] Wu L Q, Li W L, Yan F L.J Heterocyclic Chem, 2010, 47: 1246

[15] Khaligh N G, Shirini F. J Mol Catal A, 2011, 348: 20

[16] Khaligh N G. Ultrason Sonochem, 2012, 19: 736

[17] Khaligh N G. Catal Sci Technol, 2012, 2: 2211

[18] Khaligh N G. Tetrahedron Lett, 2012, 53: 1637

[19] Ghashang M, Mansoor S S, Aswin K. J Adv Res, 2013, http://dx.doi. org/10.1016/j.jare.2013.03.003

[20] Ghashang M, Aswin K, Mansoor S S. Res Chem Intermed, 2013, doi: 10.1007/s11164-013-1027-1

[21] Mansoor S S, Aswin K, Logaiya K, Sudhan S P N. Arab J Chem, 2012 , http://dx.doi.org/10.1016/j.arabjc.2012.10.017

[22] Mansoor S S, Aswin K, Logaiya K, Sudhan P N, Malik S. Res Chem Intermed, 2012, doi: 10.1007/s11164-012-0968-0

[23] Khodaei M M, Khosropour A R, Moghanian H. Synlett, 2006: 916 